\title{
ZETA FUNCTIONS OF INFINITE GRAPH BUNDLES
}

\author{
SAMUEL COOPER* AND STRATOS PRASSIDIS**
}

\begin{abstract}
We compute the equivariant zeta function for bundles over infinite graphs and for infinite covers. In particular, we give a "transfer formula" for the zeta function of infinite graph covers. Also, when the infinite cover is given as a limit of finite covers, we give a formula for the limit of the zeta functions.
\end{abstract}

\section{INTRODUCTION}

The Ihara zeta function of a finite graph reflects combinatorial and spectral properties of that graph ([13], 2], [18]). Originally, Ihara defined the zeta function on finite graphs imitating the classical definition of the zeta function:

$$
\zeta_{X}(z)=\prod_{[C]}\left(1-z^{\ell(C)}\right)^{-1}
$$

where the product is over all equivalence classes of primitive closed loops $C$ in $X$ and $\ell(C)$ denotes the length of $C$. In [2], it was shown that, for a finite graph $X$ :

$$
\zeta_{X}(z)^{-1}=\left(1-z^{2}\right)^{\epsilon-\nu} \operatorname{det}\left(I-z A+z^{2} Q\right),
$$

where $\epsilon$ is the number of edges, $\nu$ is the number of vertices, $A$ is the adjacency matrix of $X$, and $Q$ is the diagonal matrix with entries $\operatorname{deg}(v)-1$, for each $v \in V(X)$. In [8], the definition of Ihara zeta function was extended to infinite graphs that are limits of sequences of finite graphs. In particular, it was shown in [8], using the results in [17], that the sequence of the zeta functions of the finite graphs converges. In [3], 4], [10], [11, [12, the expression of the zeta function as a rational function was extended to infinite graphs that admit an action of a discrete group $\Gamma$ with finite quotient. The determinant in the finite case is replaced by the determinant in a von Neumann algebra $\mathcal{N}_{0}(X, \Gamma)$ of all the bounded operators on $L^{2}(V(X))$. In [6] the zeta function of finite graph bundles over finite was computed generalizing the results on graph coverings that appear [18, [19], [20]. Their results can be described as transfer results for the Ihara zeta function.

We combine the results on infinite graphs and bundles to derive a transfer formula for infinite bundles and coverings. Let $\phi$ be an $\operatorname{Aut}(F)$-assignment on $X$. Let $(\Gamma, \Delta)$ be a pair of groups that act on $X$ and $F$ in such a way that the actions are $\phi$-compatible and by finite co-volume.

2000 Mathematics Subject Classification. Primary 11M36; Secondary 05C50, 11M41.

* Partially supported by an NSF REU grant.

** Partially supported by Canisius College Summer Grant and an NSF REU grant. 
Theorem (Main Theorem 1). With the above assumptions, the equivariant zeta function is given by:

$\zeta_{X \times \psi} F, \Gamma \times \Delta(z)^{-1}=\left(1-z^{2}\right)^{-\chi^{(2)}\left(X \times{ }^{\psi} F\right)} \operatorname{det}_{\Gamma \times \Delta}\left(I-\sum_{\gamma \in \operatorname{Aut}(F)}\left(A_{\vec{X}_{(\psi, \gamma)}} \otimes P_{\gamma}+I_{X} \otimes A_{F}\right) z+Q z^{2}\right)$,

where $A_{\vec{X}_{(\phi, \gamma)}}$ is the adjacency matrix of the directed graph spanned by the edges in $\psi^{-1}(\gamma), P_{\gamma}$ is the permutation matrix induced by the action of $\gamma$ on $V(F), \chi^{(2)}$ is the Euler characteristic of the quotient $X_{1} \times{ }^{\phi_{1}} F$, det $t_{\Gamma \times \Delta}$ is the determinant defined on the von Neumann algebra of $\Gamma \times \Delta$, and $Q$ is the diagonal operator such that $Q(y, i)=\operatorname{deg}(y)+\operatorname{deg}(i)-1$.

Using similar methods, we prove a decomposition formula of the Ihara zeta function for infinite covers. Let $p: Y \rightarrow X$ be a cover with $X$ finite. Let $\Gamma=\operatorname{Cov}(p)$.

Theorem (Main Theorem 2). With the above notation,

$$
\zeta_{Y, \Gamma}(z)^{-1}=\left(1-z^{2}\right)^{-\chi^{(2)}(Y)} \operatorname{det}_{\Gamma}\left(I-\left(\sum_{\gamma \in \Gamma} A_{\left(\vec{Y}_{(\psi, \gamma)}\right)} \otimes P_{\gamma}\right) z+Q z^{2}\right),
$$

where $Q$ is the diagonal operator with $(x, \gamma)$-entry $\operatorname{deg}(x)-1$.

We apply the above calculations to sequences of strongly convergent graphs. In particular, a sequence $\left\{\left(X_{n}, w_{n}\right)\right\}_{n \in \mathbb{N}}$ is strongly convergent to $(X, w)$ if it is a covering sequence of regular graphs converging to $X$ in such a way that $X$ covers compatibly each element of the sequence. Such sequences appear when we consider the Cayley graphs of finite quotients of a groups converging to the Cayley graph of the group.

\section{Preliminaries}

We now define a number of terms that we will use later on.

Definition 2.1. Let $G$ be any locally finite graph. Then we define the adjacency operator $A_{G}$ as follows: for any $u, v \in V(G)$,

$$
A_{G}(u, v)= \begin{cases}1, & \text { if } u \sim v \\ 0, & \text { otherwise }\end{cases}
$$

The definition makes sense even if the graph is directed. If $G$ is undirected, the $A_{G}$ is symmetric.

Definition 2.2. Let $\tilde{G}$ and $G$ be locally finite graphs. We say that

$$
p: \tilde{G} \rightarrow G
$$

is a graph covering if the following two conditions hold:

(1) If $x \sim_{\tilde{G}} y$, then $p(x) \sim_{G} p(y)$. 
(2) For any $x \in \tilde{G}, p: N(x) \rightarrow N(p(x))$ is a bijection.

The first condition in the definition means that $p$ is a graph map. The second condition is a local triviality condition.

Graph bundles are defined in [15. They generalize the graph coverings in the sense that the "fiber graph" is allowed to have a non-empty set of edges. We will concentrate on bundles with finite fibers. For a graph $X$, we denote by $E(\vec{X})$ the set of ordered edges-i.e., each edge of $X$ appears twice, each with opposite orientation.

Definition 2.3. Let $G$ be any locally finite graph (possibly infinite), let $F$ be a finite graph. We define an $\operatorname{Aut}(F)$-voltage assignment on $G$ by

$$
\phi: E(\vec{G}) \rightarrow \operatorname{Aut}(F), \quad \phi(u v)=\phi(v u)^{-1} .
$$

Definition 2.4. Let $G$ be a locally finite graph, $F$ a finite graph, and $\phi$ an $\operatorname{Aut}(F)$-voltage assignment on $G$. We define a graph bundle $G \times{ }^{\phi} F$ to be the graph with vertex set $V(G) \times V(F)$, with two vertices $(u, i),(v, j) \in G \times \times^{\phi} F$ adjacent if either one of the following two conditions hold:

(1) $u \sim v$ and $j=i^{\phi(u v)}$

(2) $u=v$ and $i \sim j$.

Let $\phi$ be a $\operatorname{Aut}(F)$-voltage assignment on $G$. Let $\gamma \in \operatorname{Aut}(F)$.

(1) Let $\vec{G}_{(\phi, \gamma)}$ denote the spanning subgraph of the digraph $\vec{G}$ whose directed edge set is $\phi^{-1}(\gamma)$.

(2) We define the permutation operator $P_{\gamma}$ by the following formula: for any two vertices $i, j$ in $V(F)$,

$$
P_{\gamma}(i, j)= \begin{cases}1, & \text { if } j=i^{\gamma} \\ 0, & \text { otherwise. }\end{cases}
$$

Remark 2.5. When the graphs are infinite, the matrices defined above are operators on the Hilbert space with basis the vertex set of the graph. More precisely, if $G$ is any locally finite graph, set $L^{2}(G)$ to be the Hilbert space:

$$
L^{2}(G)=\left\{f: V(G) \rightarrow \mathbb{C}: \sum_{u \in V(G)}|f(u)|^{2}<\infty\right\} .
$$

Then the adjacency operator is given by

$$
A(f)(u)=\sum_{u \sim v} f(v)
$$

With the above notation,

$$
P_{\gamma}(f)(i)=f(\gamma(i))
$$

The following combines covering maps and bundles. 
Theorem 2.6. Let $F$ and $X$ be locally finite graphs. Let $X$ be equipped with an Aut $(F)$-voltage assignment $\phi$. Let $p: Y \rightarrow X$ be a covering map and $\psi$ the Aut $(F)$-voltage assignment

$$
\psi: E(\vec{Y}) \rightarrow \operatorname{Aut}(F), \quad \psi(x y)=\phi(p(x) p(y)) .
$$

Define a graph map

$$
\tilde{p}: Y \times{ }^{\psi} F \rightarrow X \times{ }^{\phi} F, \quad \tilde{p}(x, i)=(p(x), i) .
$$

Then $\tilde{p}$ is a covering map.

Proof. First we will show prove that $\tilde{p}$ is a graph map i.e., that is preserves adjacency. Let $(x, i) \sim$ $(y, j)$ in $Y \times{ }^{\psi} F$. There are two cases to consider:

(1) Suppose $x \sim y$ in $Y$. Then $p(x) \sim p(y)$, and $j=i^{\psi(x y)}=i^{\phi(p(x) p(y))}$. Thus, by definition,

$$
\tilde{p}(x, i)=(p(x), i) \sim(p(y), j)=\tilde{p}(y, j), \text { in } X \times{ }^{\phi} F .
$$

(2) Suppose $x=y$. Then $i \sim j$ in $F$, and clearly $p(x)=p(y)$. Thus, by definition, $\tilde{p}(x, i) \sim$ $\tilde{p}(y, j)$.

Thus $\tilde{p}$ preserves adjacency.

Now we must show that $\left.\tilde{p}\right|_{N(x, i)}$ is a bijection.

$\left.\tilde{p}\right|_{N(x, i)}$ is an injection. Let $\left(y_{1}, j_{1}\right),\left(y_{2}, j_{2}\right) \in N(x, i)$ with $\tilde{p}\left(y_{1}, j_{1}\right)=\tilde{p}\left(y_{2}, j_{2}\right)$. Then we know that

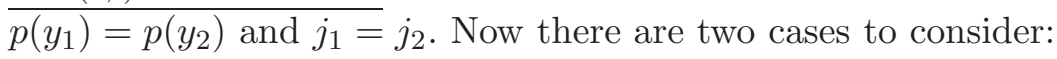

(1) Suppose $y_{1}=x$ and $i \sim j_{1}$. Then $i \sim j_{2}$, and since $(x, i) \sim\left(y_{2}, j_{2}\right)$, we must have $x=y_{2}$. Thus $y_{1}=y_{2}$, so $\left(y_{1}, j_{1}\right)=\left(y_{2}, j_{2}\right)$. The same argument works if $y_{2}=x$.

(2) Suppose $y_{1} \sim x$, and $i=j_{1} \psi\left(y_{1} x\right)=j_{1} \phi\left(p\left(y_{1}\right) p(x)\right)$. Since $p\left(y_{1}\right)=p\left(y_{2}\right)$, we see that $i=$ $j_{2} \phi\left(p\left(y_{2}\right) p(x)\right)$. Thus, since $\left(y_{2}, j_{2}\right) \sim(x, i)$, we must have $y_{2} \sim x$. Now, since $p$ is a graph covering map, $\left.p\right|_{N(x)}$ is a bijection. But $y_{1}, y_{2} \in N(x)$ and $p\left(y_{1}\right)=p\left(y_{2}\right)$; thus, $y_{2}=y_{1}$, so $\left(y_{1}, j_{1}\right)=\left(y_{2}, j_{2}\right)$.

$\left.\tilde{p}\right|_{N(x, i)}$ is a surjection. Let $(u, k) \in N((p(x), i)$. Again there are two cases to consider:

(1) Suppose $u=p(x)$ and $i \sim k$. Then $\tilde{p}(x, k)=(u, k)$, and by definition, $(x, k) \in N(x, i)$.

(2) Suppose $u \sim p(x)$ and $i=k^{\phi(u p(x))}$. Since $\left.p\right|_{N(x)}$ is a surjection, there exists some $y \in N(x)$ such that $p(y)=u$. Then $y \sim x$ and $i=k^{\phi(p(y) p(x))}=k^{\psi(y x)}$. Thus, $(y, k) \in N(x, i)$ and $\tilde{p}(y, k)=(u, k)$.

Therefore, $\left.\tilde{p}\right|_{N(x, i)}$ is a bijection. This completes the proof.

The vertex set of a bundle over $G$ is $V(G) \times V(F)$. Then

$$
L^{2}\left(G \times{ }^{\phi} F\right)=L^{2}(G) \otimes L^{2}(F)
$$


where the tensor product takes place in the category of Hilbert spaces. More precisely, it is the completion of the algebraic tensor product. The following theorem (proved in [15] for the finite case) provides a decomposition for the adjacency operator of any graph bundle.

Theorem 2.7. Let $\phi$ be an Aut $(F)$-voltage assignment on a locally finite graph $G$, with F locally finite. Then

$$
A_{G \times \phi} F=\bigoplus_{\gamma \in \operatorname{Aut}(F)} A_{\vec{G}_{(\phi, \gamma)}} \otimes P_{\gamma}+I_{G} \otimes A_{F}
$$

Proof. It is enough to prove the result for functions of the form $f \otimes g$, where $f \in L^{2}(G)$ and $g \in L^{2}(F)$. Let $(u, i) \in V\left(G \times{ }^{\phi} F\right)$. Then

$$
A_{G \times{ }^{\phi} F}(f \otimes g)(u, i)=\sum_{(u, i) \sim(v, j)} f(v) g(j) .
$$

The right hand side is given by:

$$
\bigoplus_{\gamma \in \operatorname{Aut}(F)} A_{\vec{G}_{(\phi, \gamma)}} \otimes P_{\gamma}(f \otimes g)(u, j)+I_{G} \otimes A_{F}(f \otimes g)(u, j)=\bigoplus_{\gamma \in \operatorname{Aut}(F), u \in \vec{G}_{(\phi, \gamma)}} A_{\vec{G}_{(\phi, \gamma)}}(f \mid)(u) P_{\gamma}(g)(i)+f(u) A_{F}(g)(i)
$$

There are two possibilities for $(u, i) \sim(v, j)$ :

(1) $u \sim v$ and $i=j^{\phi(u v)}$. Then the right hand side becomes:

$$
A_{\vec{G}_{(\phi, \phi(u v))}}(f \mid)(u) P_{\gamma}(g)(i)=f(v) g(j) .
$$

(2) $u=v$ and $i \sim j$. In the right hand side, only the last summand is non-zero and it is equal to $f(u) g(j)$.

Finally, it is clear that if neither $u \sim v$ nor $u=v$, then the sum on the right hand side is zero. This completes the proof.

By a marked graph, we mean a pair $(X, w)$ with $X$ a graph and $w$ a distinguished vertex.

Definition 2.8. On the space of marked graphs there is a metric dist defined as follow:

$$
\operatorname{dist}\left(\left(X_{1}, w_{1}\right),\left(X_{2}, w_{2}\right)\right)=\inf \left\{\frac{1}{n+1} ; B_{X_{1}}\left(w_{1}, n\right) \text { is isometric to } B_{X_{2}}\left(w_{2}, n\right)\right\},
$$

where $B_{X}(w, n)$ is the combinatorial ball of radius $n$ in $X$ centered on $v$.

For a sequence of marked graphs $\left\{\left(X_{n}, w_{n}\right)\right\}_{n \in \mathbb{N}}$, we say that $(X, w)$ is the limit graph if

$$
\lim _{n \rightarrow \infty} \operatorname{dist}\left((X, w),\left(X_{n}, w_{n}\right)\right)=0 .
$$

For a finite graph $X$, let $c_{r}(X)$ denote the number of closed paths in $X$ of length $r$. Let

$$
(X, w)=\lim _{n \rightarrow \infty}\left(X_{n}, w_{n}\right)
$$


where $\left\{\left(X_{n}, w_{n}\right)\right\}_{n \in \mathbb{N}}$ is a covering sequence of $k$-regular marked graph. In [8], the definition of the number $c_{r}$ is extended for the graph $X$ as follows:

$$
\tilde{c}_{r}=\lim _{n \rightarrow \infty} \frac{c_{r}\left(X_{n}\right)}{\left|X_{n}\right|}
$$

In [8], it was shown that the limit exists. The zeta function $\zeta(X, w)$ of the marked graph $X$, with respect to the sequence $\left\{\left(X_{n}, w_{n}\right)\right\}_{n \in \mathbb{N}}$, is defined by

$$
\ln \zeta_{(X, w)}(z)=\lim _{n \rightarrow \infty} \frac{1}{\left|X_{n}\right|} \ln \zeta_{X_{n}}(z)=\sum_{r=1}^{\infty} \frac{\tilde{c_{r}} z^{r}}{r}, \quad|z|<\frac{1}{k-1} .
$$

The proof that the series has a non-trivial radius of convergence is given in [8] and depends on results from [17].

Let $X$ be a graph such that the degrees of vertices is bounded. Let $\Gamma$ be a group of graph automorphisms of the graph $X$ that acts on $X$ without inversions and satisfying the following properties:

(1) For each $v \in V(X)$, the stabilizer $\Gamma_{v}=\{\gamma \in \Gamma: \gamma v=v\}$ is finite.

(2) If $\mathcal{F}_{0} \subset V(X)$ is a complete set of orbit representatives of the action of $\Gamma$ on $V(X)$, then

$$
\operatorname{vol}(X / \Gamma)=\sum_{v \in \mathcal{F}_{0}} \frac{1}{\left|\Gamma_{v}\right|}<\infty
$$

In particular, if the action of $\Gamma$ on $V(X)$ is free, the second condition is equivalent to the condition that the orbit space $V(X) / \Gamma$ is finite. In this case, the Ihara zeta function is defined as

$$
\zeta_{X, \Gamma}(z)=\prod_{C \in \mathcal{P} / \Gamma}\left(1-z^{\ell(C)}\right)^{\left|\Gamma_{C}\right|}
$$

where:

- $\mathcal{P}$ are equivalence classes of closed, primitive, tail-less edge-paths without backtracking. Two such circuits are equivalent if they differ only by a shift. $\mathcal{P} / \Gamma$ denotes the orbit space of $\mathcal{P}$ under the $\Gamma$ action.

- For each class $C \in \mathcal{P} / \Gamma, \ell(C)$ denotes the length of $C$ i.e., the number of edges in $C$.

- $\Gamma_{C}$ denotes the isotropy group of $C$.

This formula generalizes the classical zeta function on finite graphs.

We will describe the analogue of Bass' formula for $\zeta_{X, \Gamma}(z)$ Let $L^{2}(X)$ be the Hilbert space of functions on $V(X)$. A unitary representation is given by:

$$
\lambda_{0}: \Gamma \rightarrow U\left(L^{2}(X)\right), \quad\left(\lambda_{0}(\gamma) f\right)(v)=f\left(\gamma^{-1} v\right), \gamma \in \Gamma, f \in L^{2}(X), v \in V(X) .
$$

Then the von Neumann algebra of all bounded operators on $L^{2}(X)$ that commute with the $\Gamma$ action is defined as:

$$
\mathcal{N}_{0}(X, \Gamma)=\left\{\lambda_{0}(\gamma): \gamma \in \Gamma\right\}^{\prime}
$$


The algebra $\mathcal{N}_{0}(X, \Gamma)$ inherits a trace given by:

$$
\operatorname{Tr}_{\Gamma}(A)=\sum_{v \in \mathcal{F}_{0}} \frac{1}{\left|\Gamma_{v}\right|} A(v, v), \quad A \in \mathcal{N}_{0}(X, \Gamma) .
$$

With this setting, the Bass formula for the Ihara zeta function has the form ([3], [4, [10, [11, [12]):

$$
\zeta_{X, \Gamma}^{-1}(z)=\left(1-z^{2}\right)^{-\chi^{(2)}(X)} \operatorname{det}_{\Gamma}\left(\Delta_{X, z}\right),
$$

where

- $\operatorname{det}_{\Gamma}=\exp \circ \operatorname{Tr}_{\Gamma}$ oln is the determinant in the von Neumann algebra $\mathcal{N}_{0}(X, \Gamma)$.

- $\Delta_{X, z}=I-A z+Q z^{2}$, with $A$ the adjacency operator on $X$, and $Q$ is the operator on $L^{2}(X)$ given by:

$$
Q(f)(v)=(\operatorname{deg}(v)-1) f(v), \quad \text { for each } v \in V(X)
$$

\section{Remark 2.9.}

(1) In [3, 44, 10], 11], 12, it was shown that the function $\zeta_{X, \Gamma}$ is defined for sufficiently small $|u|$. More precisely, if $k$ is the maximum degree of $X, \zeta_{X, \Gamma}(u)$ is a holomorphic function for all $|u|<\frac{1}{d-1}$.

(2) $\chi^{(2)}(X)$ is the Euler characteristic defined in [3]. In most applications, it is equal to $\chi(X / \Gamma)$, the Euler characteristic of the orbit space.

(3) Let $X$ be a $k$-regular graph and $q=k-1$. Using the determinant formula, the zeta function can be extended to a holomorphic function in the open set ([3], [10]):

$$
\Omega_{q}=\mathbb{R}^{2} \backslash\left(\left\{(x, y) \in \mathbb{R}^{2}: x^{2}+y^{2}=\frac{1}{q}\right\} \bigcup\left\{(x, 0) \in \mathbb{R}^{2}: \frac{1}{q} \leq|x| \leq 1\right\}\right) .
$$

(4) In the above references there is an interpretation of the Bass formula over the determinant on $\mathcal{N}_{1}(X, \Gamma)$, the von Neumann algebra on the set of edges of $X$.

Notation. There are three types of zeta functions used in this paper.

(1) We write $\zeta_{X}(z)$ for the classical zeta function defined for a finite graph $X$.

(2) We write $\zeta_{X, \Gamma}(z)$ for the equivariant zeta function defined on an infinite graph $X$ equipped with an action of a group $\Gamma$ with finite co-volume.

(3) We write $\zeta_{(X, w)}(u)$ for the zeta function that it is the limit of $\zeta_{X_{n}}(z)^{1 /\left|V\left(X_{n}\right)\right|}$, where $\left\{\left(X_{n}, w_{n}\right)\right\}_{n \in \mathbb{N}}$ is a covering sequence of finite regular graphs converging to $(X, w)$.

Definition 2.10. The sequence $\left\{X_{n}, w_{n}\right\}_{n \in \mathbb{N}}$ strongly converges to $(X, w)$ if: 
(1) $\left\{X_{n}, w_{n}\right\}_{n \in \mathbb{N}}$ is a covering sequence of marked $k$-regular graphs with

$$
p_{m-1}: X_{m} \rightarrow X_{m-1}
$$

the covering map.

(2) $X$ is $k$-regular.

(3) There are covering maps

$$
\rho_{n}: X \rightarrow X_{n}
$$

such that:

(a) $\rho_{1}(u)=p_{1} p_{2} \ldots p_{n-1}\left(\rho_{n}(u)\right)$, for all $n$.

(b) For each $n$, the isometry between $B_{X}\left(w, s_{n}\right)$ and $B_{X_{n}}\left(w_{n}, s_{n}\right)$ is given by the restriction of $\rho_{n}$.

Remark 2.11. Cayley graphs of groups give sequences of graphs that strongly converge. Let $\Gamma$ be a group, $S$ a symmetric set of generators and $\left\{K_{n}\right\}_{n \in \mathbb{N}}$ a sequence of normal subgroups of finite index such that:

$$
K_{1} \supset K_{2} \supset K_{3} \ldots, \text { and } \bigcap_{n \in \mathbb{N}} K_{n}=\{K\} .
$$

Then the sequence of the marked Schreier graphs $\left\{\left(\mathcal{S}\left(\Gamma, K_{n}, S\right), 1\right)\right\}_{n \in \mathbb{N}}$ strongly converges to $(\mathcal{S}(\Gamma, K, S), 1)$.

Let $\left\{X_{n}, w_{n}\right\}_{n \in \mathbb{N}}$ strongly converge to $(X, w)$ and $\Gamma_{n}=\operatorname{Cov}\left(X, X_{n}\right)$. The next results gives a connection between the different types of zeta functions.

Theorem 2.12. Assume that all the graphs in the sequence are $k$-regular finite graphs converging to a k-regular graph $(X, w)$. Then

$$
\lim _{n \rightarrow \infty} \zeta_{X_{n}}(z)^{\frac{\left|V\left(X_{1}\right)\right|}{\left|V\left(X_{n}\right)\right|}}=\zeta_{X, \Gamma_{1}}(z)=\zeta_{(X, w)}(z)^{\left|V\left(X_{1}\right)\right|} .
$$

Proof. By [4], Theorem 2.1,

$$
\lim _{n \rightarrow \infty} \zeta_{X_{n}}(z)^{\frac{1}{\left|N_{n}\right|}}=\zeta_{X, \Gamma_{1}}(z)
$$

where $N_{n}=\left[\Gamma_{n}, \Gamma_{1}\right]=\left|V\left(X_{n}\right)\right| /\left|V\left(X_{1}\right)\right|$. The result follows from the definition of $\zeta_{(X, w)}(z)$.

The following is the main part of the proof of Theorem 2.1 in [4].

Corollary 2.13. With the above notation,

$$
\operatorname{det}_{\Gamma_{1}}\left(\Delta_{X, z}\right)=\lim _{n \rightarrow \infty}\left(\Delta_{X_{n}, z}\right)^{\frac{\left|V\left(X_{1}\right)\right|}{\left|V\left(X_{n}\right)\right|}}
$$

Let $F$ be a locally finite graph and $\phi_{1}$ an $\operatorname{Aut}(F)$-voltage assignment on $X_{1}$. Inductively, define an $\operatorname{Aut}(F)$-voltage assignment on $X_{n}$ by:

$$
\phi_{n}(u v)=\phi_{n-1}\left(p_{n-1}(u) p_{n-1}(v)\right) .
$$


Also, define an Aut $(F)$-voltage assignment on $X$ by:

$$
\phi(u v)=\phi_{1}\left(\rho_{1}(u) \rho_{1}(v)\right)
$$

The details are presented in the following diagram.

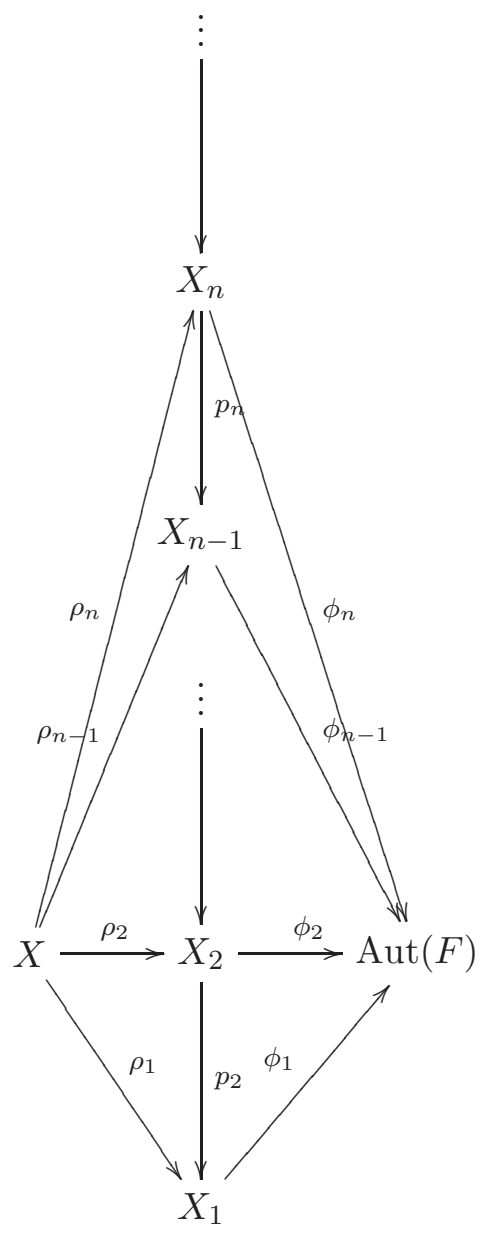

Now, by Theorem 2.6, we know that, for any finite, $d$-regular graph $F$, the sequence $\left\{X_{n} \times{ }^{\phi_{n}}\right.$ $F\}_{n \in \mathbb{N}}$ is a $k d$-regular covering sequence; thus, by [8], it converges. We will show that in fact it converges to the graph $X \times{ }^{\phi} F$. To do this will need the following:

Lemma 2.14. Assume that $\left\{\left(X_{n}, w_{n}\right)\right\}_{n \in \mathbb{N}}$ strongly converges to $(X, w)$. Then

$$
\widetilde{\rho_{n}}: B_{X \times{ }^{\phi} F}\left((w, i), s_{n}\right) \rightarrow B_{X_{n} \times \phi_{n} F}\left(\left(w_{n}, i\right), s_{n}\right), \quad \widetilde{\rho_{n}}(u, i)=\left(\left(\rho_{n}(u), i\right),\right.
$$

is an isometry, for any $i \in V(F)$ and for all $n \in \mathbb{N}$. 
Proof. Since $\rho_{n}$ is a bijection, it is clear that $\widetilde{\rho_{n}}$ is a bijection; thus, we must show that $\widetilde{\rho_{n}}$ preserves adjacency. To this end, assume $(u, i) \sim(v, j)$, for $(u, i),(v, j) \in B_{X \times{ }^{\phi} F}(w, i)$. Then there are two cases:

(1) $u \sim v$ and $j=i^{\phi(u v)}$

(2) $u=v$ and $i \sim j$.

In the case where $u=v$, since clearly $\rho_{n}(u)=\rho_{n}(v)$, we must have $\widetilde{\rho_{n}}(u, i) \sim \widetilde{\rho_{n}}(v, j)$. In the case where $u \sim v$, we must have $\rho_{n}(u) \sim \rho_{n}(v)$. Thus we must show that

$$
j=i^{\phi_{n}\left(\rho_{n}(u) \rho_{n}(v)\right)} .
$$

Now, by the definition of $\phi$,

$$
\phi(u v)=\phi_{1}\left(\rho_{1}(u) \rho_{1}(v)\right),
$$

and by assumption,

$$
\phi_{1}\left(\rho_{1}(u) \rho_{1}(v)\right)=\phi_{1}\left(p_{n-1} \ldots p_{1}\left(\rho_{n}(u)\right), p_{n-1} \ldots p_{1}\left(\rho_{n}(v)\right)\right) .
$$

But by the definition of $\phi_{n}$,

$$
\phi_{n}\left(\rho_{n}(u) \rho_{n}(v)\right)=\phi_{1}\left(p_{n-1} \ldots p_{1}\left(\rho_{n}(u)\right), p_{n-1} \ldots p_{1}\left(\rho_{n}(v)\right)\right) .
$$

This shows that

$$
\phi_{n}\left(\rho_{n}(u) \rho_{n}(v)\right)=\phi(u v)
$$

and thus

$$
j=i^{\phi(u v)} \Longrightarrow j=i^{\phi_{n}\left(\rho_{n}(u) \rho_{n}(v)\right)} .
$$

This shows that $\hat{\rho_{n}}$ preserves adjacency, and thus is an isometry. This completes the proof.

As a corollary, we have the following theorem.

Theorem 2.15. For each $i \in F$, the covering sequence $\left\{\left(X_{n} \times{ }^{\phi_{n}} F,\left(w_{n}, i\right)\right)\right\}_{n \in \mathbb{N}}$ strongly converges to $\left(X \times^{\phi} F,(w, i)\right)$.

Proof. Theorem 2.6 implies that the covering conditions of the strong convergence are satisfied The rest of the proof follows from Lemma 2.14 and [8].

\section{Zeta Functions for Bundles and Coverings}

In this section we will use our previous result and [3], 4], 10], [11, [12, to generalize the results of [6] to infinite graph bundles.

Definition 3.1. Let $X$ be a graph equipped with an $\operatorname{Aut}(F)$-voltage assignment $\phi$.

(1) An action of a group $\Gamma$ on $X$ without edge inversions is called $(F, \phi)$-compatible if

$$
\phi(\gamma(u) \gamma(v))=\phi(u v), \text { for all } u, v \in V(X), \gamma \in \Gamma \text {. }
$$


(2) An action of a group $\Delta$ without inversions on $F$ is called $(X, \phi)$-compatible if $\operatorname{Im}(\phi) \subset$ $C_{\text {Aut }(F)}(\Delta)$ i.e., the image of $\phi$ centralizes $\Delta$.

(3) The pair of groups $(\Gamma, \Delta)$ as before is called $\phi$-compatible if $\Gamma$ is $(F, \phi)$-compatible and $\Delta$ is $(X, \phi)$-compatible.

Lemma 3.2. With the above notation, if the pair $(\Gamma, \Delta)$ is $\phi$-compatible, then the product action:

$$
(\gamma, \delta)(x, i)=(\gamma x, \delta i), \quad(\gamma, \delta) \in \Gamma \times \Delta, \quad(x, i) \in V\left(X \times{ }^{\phi} F\right),
$$

is an action by graph automorphisms on $X \times{ }^{\phi} F$. Furthermore, if the action of $\Gamma$ and $\Delta$ are of finite co-volume, so is the action of $\Gamma \times \Delta$ on $X \times{ }^{\phi} F$.

Proof. The proof follows from the definitions.

Theorem 3.3. Assume that $(\Gamma, \Delta)$ is a pair of $\phi$-compatible actions. Also, assume that the actions are of finite co-volume. Then

$$
\zeta_{X \times \phi} F, \Gamma \times \Delta(z)^{-1}=\left(1-z^{2}\right)^{-\chi^{(2)}\left(X \times^{\phi} F\right)} \operatorname{det}_{\Gamma \times \Delta}\left(I-\sum_{\gamma \in \operatorname{Aut}(F)}\left(A_{\vec{X}_{(\phi, \gamma)}} \otimes P_{\gamma}+I_{X} \otimes A_{F}\right) z+Q z^{2}\right),
$$

$\chi^{(2)}$ is the Euler characteristic and $Q$ is the diagonal operator with $(x, i)$-entry $\operatorname{deg}(x)+\operatorname{deg}(i)-1$. Furthermore, the zeta function is holomorphic for $|z|<\frac{1}{k+d-1}$. If $X$ is $k$ regular and $F d$-regular, then $\zeta_{X \times{ }^{\phi} F, \Gamma}(z)$ can be extended to a holomorphic function on $\Omega_{k+d-1}$.

Proof. From [10], 11], we have

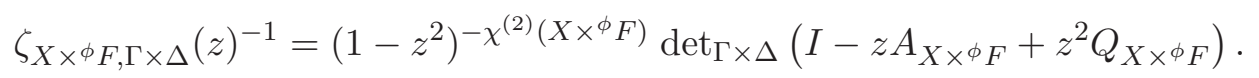

The theorem now follows immediately from Theorem 2.7 .

We will now use Theorem 3.3 to provide a decomposition for the zeta function of any infinite cover. Let $p: Y \rightarrow X$ be a cover with $X$ finite and $Y$ locally finite. Let $\operatorname{Cov}(p)=\Gamma$. Now we define the function

$$
\phi: E(\vec{X}) \rightarrow \Gamma \text {. }
$$

For this we write $X=\left\{x_{1}, \ldots x_{n}\right\}$. For each $i$, choose $v_{i} \in Y$ such that $p\left(v_{i}\right)=x_{i}$. Now, since $p: N\left(v_{i}\right) \rightarrow N\left(x_{i}\right)$ is a bijection, for each $x_{j} \in N\left(x_{i}\right)$ there exists a unique $u_{j} \in N\left(v_{i}\right)$ such that $p\left(u_{j}\right)=x_{j}$. So, since $p\left(v_{j}\right)=x_{j}=p\left(u_{j}\right)$, there exists some $\gamma \in \Gamma$ such that $\gamma v_{j}=u_{j}$. Thus, define

$$
\phi: E(\vec{X}) \rightarrow \Gamma, \quad \phi\left(x_{i} x_{j}\right)=\gamma .
$$

We then have the following:

Lemma 3.4. Let $\phi$ be defined as above. Then

(1) The $\Gamma$ action on $Y$ is of finite co-volume and it is $\phi$-compatible. 
(2) The map

$$
\alpha: Y \rightarrow X \times^{\phi} \Gamma, \quad \alpha(u)=(p(u), \beta)
$$

is an isomorphism, where $\beta u=v_{i}, \quad p(u)=x_{i}=p\left(v_{i}\right)$.

Proof. The proof is folklore.

Now, in order to prove an analogue of 2.7 for $Y \simeq X \times^{\phi} \Gamma_{1}$, we need to define the following operator: for $\gamma_{1}, \gamma_{2}, \gamma \in \Gamma_{1}$,

$$
P_{\gamma}\left(\gamma_{1}, \gamma_{2}\right)= \begin{cases}1, & \text { if } \gamma_{2}=\gamma_{1} \gamma \\ 0, & \text { otherwise }\end{cases}
$$

Lemma 3.5. Let $\phi$ be defined as above. Then

$$
A_{X \times \phi}=\sum_{\gamma \in \Gamma} A_{\vec{X}_{(\phi, \gamma)}} \otimes P_{\gamma}
$$

Proof. The proof is analogous to that of 2.7

The following theorem provides a decomposition for the zeta function of any infinite cover.

Theorem 3.6. With the above notation,

$$
\zeta_{Y, \Gamma}(z)^{-1}=\left(1-z^{2}\right)^{-\chi^{(2)}(X)} \operatorname{det}_{\Gamma}\left(I-\left(\sum_{\gamma \in \Gamma} A_{\left(\vec{X}_{(\phi, \gamma)}\right)} \otimes P_{\gamma}\right) z+Q z^{2}\right),
$$

where $Q$ is the diagonal operator with $(x, \gamma)$-entry deg $(x)-1$. The function is holomorphic for $|z|<\frac{1}{k-1}$. If $Y$ is k-regular then $\zeta_{Y, \Gamma}(z)$ is holomorphic on $\Omega_{k-1}$.

Proof. This follows immediately from 3.3 and 3.4 .

Let $\left\{\left(X_{n}, w_{n}\right)_{n \in \mathbb{N}}\right.$ be a sequence of finite $k$-regular marked graphs that strongly converges to the $k$-regular marked graph $(X, w)$. Let $F$ be a finite $d$-regular graph. With the notation as in Theorem 2.15, we know that

We write $a_{n}=\left|V\left(X_{n}\right)\right|$ and $f=|F|$.

Corollary 3.7. With the above notation, for $|z|<\frac{1}{k+d-1}$,

$$
\begin{aligned}
& \zeta_{\left(X \times{ }^{\phi} F,(w, i)\right)}(z)^{-1}= \\
& =\lim _{n \rightarrow \infty}\left[\left(1-z^{2}\right)^{-\chi\left(X_{n} \times \phi_{n} F\right)} \operatorname{det}\left(I-\sum_{\gamma \in \operatorname{Aut}(F)}\left(A_{\vec{X}_{n}\left(\phi_{n}, \gamma\right)} \otimes P_{\gamma}+I_{X_{n}} \otimes A_{F}\right) z+Q_{n} z^{2}\right)\right]^{\frac{1}{f a_{n}}} \\
& =\left[\left(1-z^{2}\right)^{-\chi^{(2)}\left(X \times^{\phi} F\right)} \operatorname{det}_{\Gamma}\left(I-\sum_{\gamma \in \operatorname{Aut}(F)}\left(A_{\vec{X}_{(\phi, \gamma)}} \otimes P_{\gamma}+I_{X} \otimes A_{F}\right) z+Q z^{2}\right)\right]^{\frac{1}{f a_{1}}}
\end{aligned}
$$


Proof. The first identity follows because of Theorem 2.15:

$$
\begin{aligned}
& \zeta_{\left(X \times{ }^{\phi} F,(w, i)\right)}(z)^{-1}=\lim _{n \rightarrow \infty} \zeta_{X_{n} \times \phi_{n} F}(z)^{-\frac{1}{f a_{n}}} \\
& =\lim _{n \rightarrow \infty}\left[\left(1-z^{2}\right)^{-\chi\left(X_{n} \times{ }^{\phi_{n}} F\right)} \operatorname{det}\left(I-\sum_{\gamma \in \operatorname{Aut}(F)}\left(A_{\vec{X}_{n}\left(\phi_{n}, \gamma\right)} \otimes P_{\gamma}+I_{X_{n}} \otimes A_{F}\right) z+Q_{n} z^{2}\right)\right]^{\frac{1}{f a_{n}}}
\end{aligned}
$$

The second identity follows from Theorem 3.3 and Theorem 2.12 .

\section{Application}

Let $p: Y \rightarrow X$ be a cover with $\operatorname{Cov}(p)=\Gamma$ and $X$ a finite graph. let $F$ be a finite $d$-regular graph with $n$ such that $\operatorname{Aut}(F)$ contains a the dihedral group $D_{2 n}$ of order $2 n$. Let $\phi$ be an Aut $(F)$ voltage assignment on $X$ whose image is contained into $D_{2 n}$ and $\psi$ the induced $\operatorname{Aut}(F)$-voltage assignment on $Y$ (Theorem 2.6). By Theorem 2.6, the induced map

$$
\tilde{p}: Y \times{ }^{\psi} F \rightarrow X \times{ }^{\phi} F, \quad \tilde{p}(x, i)=(p(x), i)
$$

is a covering map. Also, $\operatorname{Cov}(\tilde{p})=\Gamma$.

The following is the setup (for the finite case this is the same as in [14] and [6]): set $V(F)=$ $\{1,2, \ldots, n\}$ and $S_{n}$ the symmetric group on $V(F)$. Let $a=\left(\begin{array}{ll}1 & 2 \ldots n-1 n\end{array}\right)$ be an $n$-cycle and let

$$
b= \begin{cases}(1 n)(2 n-1) \ldots\left(\frac{n-1}{2} \frac{n+3}{2}\right)\left(\frac{n+1}{2}\right) & \text { if } n \text { is odd } \\ (1 n)(2 n-1) \ldots\left(\frac{n}{2} \frac{n+2}{2}\right) & \text { if } n \text { is even }\end{cases}
$$

be a permutation in $S_{n}$. The permutations $a$ and $b$ generate the dihedral subgroup $D_{n}$ of $S_{n}$ :

$$
D_{n}=\left\langle a, b \mid a^{n}=b^{2}=1, b a b=a^{-1}\right\rangle .
$$

Let $\mu=\exp (2 \pi i / n)$ and $\mathbf{x}_{k}=\left(1, \mu^{k}, \mu^{2 k}, \ldots, \mu^{(n-1) k}\right)^{T}$ be the column vector in $\mathbb{C}^{n}$. Then $1, \mu$, $\ldots, \mu^{n-1}$ are the distinct eigenvalues of the permutation matrix $P(a)$ and $\mathbf{x}_{k}$ is the eigenvector corresponding to the eigenvalue $\mu^{k}$. Let $P(b)$ be the permutation matrix of $b$ and

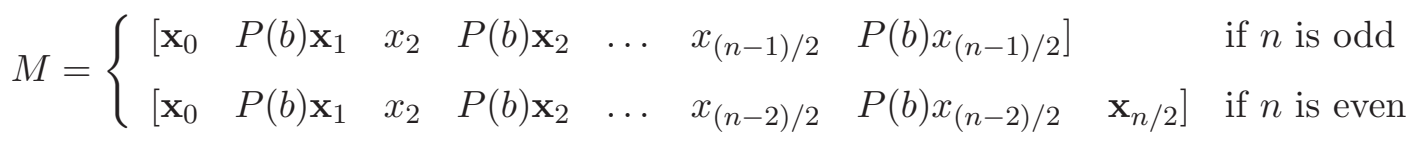

In [14, (also [6]) it was shown that $P(b) \mathbf{x}_{k}$ is an eigenvector of $P(a)$ associated with the eigenvalue $\mu^{n-k}$. Thus $M$ is invertible. Also, $P(a)$ and $A_{F}$ commute and thus they are simultaneously diagonalizable. Also, $\mathbf{x}_{k}$ and $P(b) \mathbf{x}_{k}(1 \leq k \leq(n-1) / 2$ when $n$ is odd and $1 \leq k \leq(n-2) / 2$ when $n$ is even) are eigenvectors of $A_{F}$ with the same eigenvalue of $P(b)$, denoted $\lambda_{(F, k)}$ Also, $\mathbf{x}_{0}$ is 
the eigenvector of $A_{F}$ corresponding to the eigenvalue $d$ and, for $n$ even, $\lambda_{(F, n / 2)}$ is the eigenvalue associated to the eigenvector $\mathbf{x}_{2}$. Then as in [14, using Theorem 2.7, we get that

$$
\begin{aligned}
& \left(I_{Y} \otimes M\right)^{-1} A_{Y \times{ }_{F}}\left(I_{Y} \otimes M\right)= \\
& = \begin{cases}\left(A_{Y}+d I_{Y}\right) \oplus\left(\bigoplus_{i=1}^{(n-1) / 2}\left(A_{t}+\lambda_{(F, t)}\left(I_{Y} \oplus I_{Y}\right)\right)\right. & \text { if } n \text { is odd } \\
\left(A_{Y}+d I_{Y}\right) \oplus\left(\bigoplus _ { i = 1 } ^ { ( n - 2 ) / 2 } \left(A_{t}+\lambda_{(F, t)}\left(I_{Y} \oplus I_{Y}\right) \oplus\left(B+\lambda_{(F, n / 2)} I_{Y}\right)\right.\right. & \text { if } n \text { is even }\end{cases}
\end{aligned}
$$

where

$$
B=\sum_{k=0}^{n-1}\left((-1)^{k} A\left(\vec{Y}_{\left(\psi, a^{k}\right)}\right)+(-1)^{k+1} A\left(\vec{Y}_{\left(\psi, a^{k} b\right)}\right)\right),
$$

and

$$
A_{t}=\sum_{k=0}^{n-1}\left[\begin{array}{ll}
\mu^{t k} A\left(\vec{Y}_{\left(\psi, a^{k}\right)}\right) & \mu^{t k} A\left(\vec{Y}_{\left(\psi, a^{k} b\right)}\right) \\
\mu^{(n-t) k} A\left(\vec{Y}_{\left(\psi, a^{k} b\right)}\right) & \mu^{(n-t) k} A\left(\vec{Y}_{\left(\psi, a^{k}\right)}\right)
\end{array}\right]
$$

Also, let $L_{Y}=\left(Q_{Y}+d I_{Y}\right) \otimes I_{2}$. Then the calculation in section 4 in [6] can be carried through in our setting and we get the following:

Theorem 4.1. Let $p: Y \rightarrow X$ be as above. Then

$$
\zeta_{Y \times \psi}{ }_{F, \Gamma}(z)^{-1}=\left(1-z^{2}\right)^{-\chi^{(2)}\left(Y \times{ }^{\psi} F\right)} f_{Y, F}(z) \prod_{t=1}^{(n-1) / 2} g_{Y, F, t}(z)
$$

when $n$ is odd, and

$$
\zeta_{Y \times \psi}{ }_{F, \Gamma}(z)^{-1}=\left(1-z^{2}\right)^{-\chi^{(2)}\left(Y \times{ }^{\psi} F\right)} f_{X, F}(z) h_{Y, F}(z) \prod_{t=1}^{(n-2) / 2} g_{Y, F, t}(z)
$$

when $n$ is even, where

(1) $g_{Y, F, t}(z)=\operatorname{det}_{\Gamma}\left(I_{Y} \oplus I_{Y}-\left(A_{t}+\lambda_{(F, t)}\left(I_{Y} \oplus I_{Y}\right)\right) z+L_{Y} z^{2}\right)$

(2) $h_{Y, F}(z)=\operatorname{det}_{\Gamma}\left(I_{Y}-\left(B+\lambda_{(F, n / 2)} I_{Y}\right) z+\left(Q_{Y}+d I_{Y}\right) z^{2}\right)$

(3) $f_{X, F}(z)=\operatorname{det}_{\Gamma}\left(I_{Y}-\left(A_{Y}+d I_{Y}\right) z+\left(Q_{Y}+d I_{Y}\right) z^{2}\right)$

Proof. This follows by simple calculation from 3.3 with $\Delta=\{1\}$, and Theorem 9 of [6].

Let $\left\{\left(X_{m}, w_{m}\right)\right\}_{m \in \mathbb{N}}$ be a sequence of finite regular graphs that strongly converges to $(X, w)$. Let $F$ be a finite $d$-regular with $n$ vertices such that $\operatorname{Aut}(F)$ contains $D_{2 n}$. Let $\phi$ be an $\operatorname{Aut}(F)$-voltage assignment on $X_{1}$ whose image is contained in $D_{2 n}$. Let $\phi_{n}$ be the induced Aut $(F)$-voltage on $X_{m}$ and $\psi$ be the induced $\operatorname{Aut}(F)$-voltage assignment on $X$. Set

$$
\Gamma=\operatorname{Cov}\left(X \rightarrow X_{1}\right), \quad \Delta_{m}=\operatorname{Cov}\left(X_{m} \rightarrow X_{1}\right), \quad m \in \mathbb{N} .
$$

Corollary 4.2. Let $a_{m}=\left|V\left(X_{m}\right)\right|$. With the above notation, 
(1) If $n$ is odd:

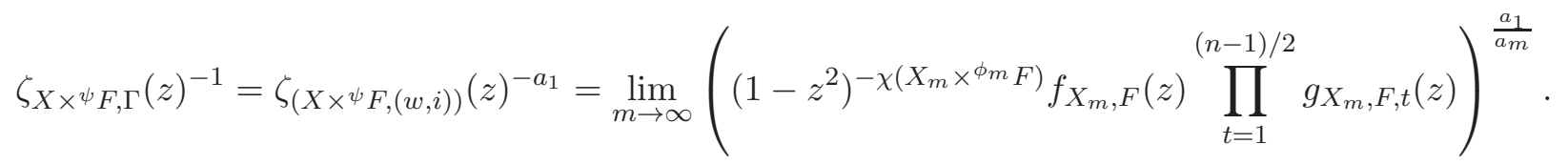

(2) If $n$ is even

$\left.\zeta_{X \times \psi_{F, \Gamma}}(z)^{-1}=\zeta_{(X \times \psi} F,(w, i)\right)(z)^{-a_{1}}=\lim _{m \rightarrow \infty}\left(\left(1-z^{2}\right)^{-\chi\left(X_{m} \times{ }^{\phi_{m}} F\right)} f_{X_{m}, F}(z) h_{X_{m}, F}(z) \prod_{t=1}^{(n-1) / 2} g_{X_{m}, F, t}(z)\right)^{\frac{a_{1}}{a_{m}}}$.

Proof. It follows from Theorem 2.12, Theorem 2.15 and Theorem 4.1.

In some cases, we can get a better description of the functions appearing in the expression for the zeta function of the limit. Assume that all the graphs $X_{m}, m \in \mathbb{N}$, and $X$ are $p$-regular. Following [8], for each $m \in \mathbb{N}$ set:

$$
\sigma_{m}=\sum \frac{\delta_{\lambda_{i}\left(X_{m}\right)}}{a_{m}}
$$

where $\lambda) i\left(X_{m}\right)$ are the eigenvalues of the Markov operator $(1 / k) A_{X_{m}}$ on $X_{m}$ and $\delta_{x}$ is the Dirac function. The sequence $\left\{\sigma_{m}\right\}_{m \in \mathbb{N}}$ weakly converges to the spectral measure $\sigma$ associated to the Markov operator $(1 / k) A_{X}$. Using the calculation of Section 5 in [8] and Corollary 2.13, we get:

$$
\begin{aligned}
\ln f_{X, F}(z) & =\operatorname{lndet}_{\Gamma}\left(I_{Y}-\left(A_{Y}+d I_{Y}\right) z+\left(Q_{Y}+d I_{Y}\right) z^{2}\right) \\
& =\lim _{m \rightarrow \infty} \frac{1}{a_{m}} \operatorname{lndet}\left(I_{X_{m}}-\left(A_{X_{m}}+d I_{X_{m}}\right) z+\left(Q_{X_{m}}+d I_{X_{m}}\right) z^{2}\right) \\
& =\lim _{m \rightarrow \infty} \int_{-1}^{1} \ln \left(1-(p \lambda+d) z+(p-1+d) z^{2}\right) d \sigma_{m}(\lambda) \\
& =\int_{-1}^{1} \ln \left(1-(p \lambda+d) z+(p-1+d) z^{2}\right) d \sigma(\lambda)
\end{aligned}
$$

where $\sigma$ is the spectral measure associated to $(1 / p) A_{X}$.

Summarizing:

Corollary 4.3. With the above notation,

$$
\ln f_{X, F}(z)=\int_{-1}^{1} \ln \left(1-(p \lambda+d) z+(p-1+d) z^{2}\right) d \sigma(\lambda), \text { for }|z|<\frac{1}{p+d-1},
$$

where $\sigma$ is the spectral measure associated to the regular random walk on $X$.

We give a specific example. The same method works for any group for which the spectral measure is known. Let $\Gamma$ be the Grigorchuk group ([1], [5, [7], [8]). Then $\Gamma$ can be represented as a subgroup of automorphisms of the rooted binary tree. Let $P=\operatorname{St}\left(1^{\infty}\right)$ be the stabilizer of the 
infinite sequence of 1 's. Let $P_{m}$ be the stabilizer of all the elements that start with $m 1$ 's and it has finite index in $\Gamma$. Then

$$
P=\bigcup_{m=1}^{\infty} P_{m}
$$

If $S=\{a, b, c, d\}$ be the standard set of generators of $\Gamma$, then the Schreier graphs $\left\{\mathbb{S}_{m}=\mathcal{S}\left(\Gamma, P_{m}, S\right)\right\}_{m \in \mathbb{N}}$ converge to $\mathbb{S}=\mathcal{S}(\Gamma, P, S)$. All the graphs have as the base point the identity coset. Then in [8], Corollary 9.2 , we have that

$$
\begin{aligned}
\ln \zeta_{\mathbb{S}, P}(z) & =-3 \ln \left(1-z^{2}\right)-\int_{-1 / 2}^{0} \frac{\left(1-8 x z+7 z^{2}\right)|1-4 x|}{2 \pi \sqrt{x(2 x-1)(2 x+1)(1-x)}} d x \\
& -\int_{1 / 2}^{1} \frac{\left(1-8 x z+7 z^{2}\right)|1-4 x|}{2 \pi \sqrt{x(2 x-1)(2 x+1)(1-x)}} d x
\end{aligned}
$$

Let $F$ be a $d$-regular graph as in the beginning of the section and $\phi$ an $\operatorname{Aut}(F)$-voltage assignment whose image lies into $D_{2 n}$. Combining Theorem 4.1 and Corollary 4.3 we get.

Theorem 4.4. With the above notation, let $\psi$ be the Aut $(F)$-voltage assignment on $\mathbb{S}$ and $R=$ $P_{1} / P$. Then

$$
\begin{aligned}
& \left.\ln \zeta_{(\mathbb{S} \times \psi} F,(P, i)\right) \\
& =\frac{1}{d a_{1}}\left[-\chi^{(2)}\left(\mathbb{S} \times{ }^{\phi} F\right) \ln \left(1-z^{2}\right)+\operatorname{lndet}_{R}\left(I-\sum_{\gamma \in \operatorname{Aut}(F)}\left(A\left(\overrightarrow{\mathbb{S}}_{(\psi, \gamma)}\right) \otimes P_{\gamma}+I_{\mathbb{S}} \otimes A_{F}\right) z+Q z^{2}\right)\right]
\end{aligned}
$$

Furthermore,

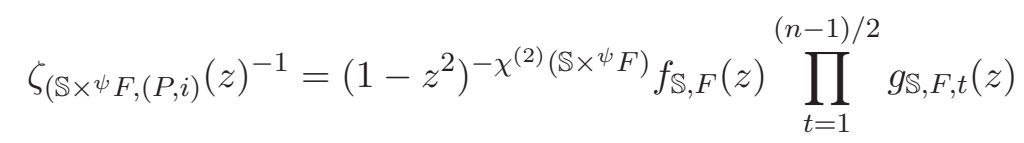

when $n$ is odd, and

$$
\zeta_{\left(\mathbb{S} \times \psi_{F,(P, i)}\right.}(z)^{-1}=\left(1-z^{2}\right)^{-\chi^{(2)}\left(\mathbb{S} \times{ }^{\psi} F\right)} f_{X, F}(z) h_{\mathbb{S}, F}(z) \prod_{t=1}^{(n-2) / 2} g_{\mathbb{S}, F, t}(z)
$$

when $n$ is even, where $g$ and $h$ are as in Theorem 4.1 and

$\ln f_{\mathbb{S}, F}(z)=\int_{-\frac{1}{2}}^{0} \frac{\ln \left(1-(8 x+d) z+(7+d) z^{2}\right)|1-4 x|}{2 \pi \sqrt{x(2 x-1)(2 x+1)(1-x)}} d x+\int_{\frac{1}{2}}^{1} \frac{\ln \left(1-(8 x+d) z+(7+d) z^{2}\right)|1-4 x|}{2 \pi \sqrt{x(2 x-1)(2 x+1)(1-x)}} d x$

\section{REFERENCES}

[1] L. Bartholdi, R. I. Grigorchuk, On the spectrum of Hecke type operators related to some fractal groups, Proc. Steklov Inst. Math., 231 (2000), 1-41.

[2] H. Bass, The Ihara-Selberg zeta function of a tree lattice, Internat. J. Math. 3 (1992), 717-797.

[3] B. Clair, S. Mokhtari-Sharghi, Zeta functions of discrete groups acting on trees, J. Algebra 237 (2001), 591-430.

[4] B. Clair, S. Mokhtari-Sharghi, Convergence of zeta functions of graphs, Proc. Amer. Math. Soc. 130 (2002), 1881-1886. 
[5] P. de la Harpe, Topics in geometric group theory, Chicago Lectures in Mathematics. University of Chicago Press, Chicago, IL, 2000.

[6] R. Feng, J. Kwak, K. Kim, Zeta functions of graph bundles, preprint.

[7] R. I. Grigorchuk, V. V. Nekrashevich, V. I. Sushchanskiı̌, Automata, dynamical systems, and groups, Proc. Steklov Inst. Math., 231 (2000), 128-203.

[8] R. Grigorchuk, A. Žuk, The Ihara zeta function of infinite graphs, the KNS spectral measure and integrable maps, in: "Random Walks and Geometry", Proc. Workshop (Vienna 2001), V. A. Kaimanovich et al., eds., de Gruyter, Berlin 2004, 141-180.

[9] R. Grigorchuk, A. Žuk, On the asymptotic spectrum of random walks on infinite families of graphs, Random walks and discrete potential theory (Cortona, 1997), 188-204, Sympos. Math., XXXIX, Cambridge Univ. Press, Cambridge, 1999.

[10] D. Guido, T. Isola, M. L. Lapidus, Ihara zeta functions for periodic simple graphs, arXiv:math.OA/0605753, May 2006.

[11] D. Guido, T. Isola, M. L. Lapidus, Ihara zeta functions for periodic simple graphs and its approximation in the amenable case, arXiv:math.OA/0608229, August 2006.

[12] D. Guido, T. Isola, M. L. Lapidus, A trace on fractal graphs and the Ihara zeta function, arXiv:math.OA/0608060, August 2006.

[13] Y. Ihara, On discrete subgroups of the two by two projective linear group over p-adic fields, J. Math. Soc. Japan 18 (1966), 219-235.

[14] J. H. Kwak, Y. S. Kwon, Characteristic polynomials of graph bundles having voltages in a dihedral group, Linear Algebra Appl. 336 (2001), 99-118.

[15] J. H. Kwak, J. Lee, Characteristic polynomials of some graph bundles II, Linear and Multilinear Algebra 32 (1992), 61-73.

[16] A. Lubotzky, Discrete Groups, Expander Graphs and Invariant Measures

[17] J.-P. Serre, Répartitions asymptotique des valeurs propres de l'opérateur de Hecke $T_{p}$, J. Amer. Math. Soc. 10 (1997), 75-102.

[18] H. M. Stark, A. A. Terras, Zeta functions of finite graphs and coverings, Adv. Math. 121 (1996), $126-165$.

[19] H. M. Stark, A. A. Terras, Zeta functions of finite graphs and coverings II, Adv. Math. 154 (2000), $132-195$.

[20] H. M. Stark, A. A. Terras, Zeta functions of finite graphs and coverings III, Adv. Math.

Department of Mathematics, Vanderbilt University, Nashville, TN 37240

E-mail address: samuel.d.cooper@vanderbilt.edu

Department of Mathematics Canisius College, Buffalo, NY 14208

E-mail address: prasside@canisius.edu 